\title{
Characterization of core-shell microstructure and self-healing performance of electrospun fiber coatings
}

Thu Q. Doan ${ }^{\mathrm{a}, \mathrm{e}}$, L. Suzanne Leslie ${ }^{\mathrm{b}, \mathrm{e}}$, Sang Yup Kim ${ }^{\mathrm{c}, \mathrm{e}}$, Rohit Bhargava ${ }^{\mathrm{b}, \mathrm{e}}$, Scott R. White ${ }^{\mathrm{d}, \mathrm{e}}$, Nancy R. Sottos ${ }^{\mathrm{a}, \mathrm{e}, *}$

${ }^{a}$ Department of Materials Science and Engineering

University of Illinois at Urbana-Champaign

1304 West Green Street

Urbana, IL, 61801, USA

${ }^{\mathrm{b}}$ Department of Bioengineering

University of Illinois at Urbana-Champaign

1304 West Springfield Avenue

Urbana, IL, 61801, USA

${ }^{c}$ Department of Mechanical Science and Engineering

University of Illinois at Urbana-Champaign

1206 W. Green Street

Urbana, IL, 61801, USA

${ }^{\mathrm{d}}$ Department of Aerospace Engineering

University of Illinois at Urbana-Champaign

104 South Wright Street

Urbana, IL, 61801, USA

${ }^{\mathrm{e}}$ Beckman Institute for Advanced Science and Technology

University of Illinois at Urbana-Champaign

405 North Mathews Avenue

Urbana, IL, 61801, USA

*Corresponding Author: Department of Materials Science and Engineering, University of Illinois at Urbana-Champaign, 1304 West Green Street, Urbana, IL, 61801, USA.

Tel.: +1 217333 1041; fax: +1 2173332736

Email address: n-sottos@illinois.edu (N.R. Sottos).

Keywords: self-healing, electrospinning, coating, encapsulation, core-shell fibers, corrosion 


\begin{abstract}
Electrospun fibers are a promising method for encapsulation of reactive agents in selfhealing coatings. Healing is initiated by mechanical damage to the coating causing the fibers to rupture and release their core materials into the damage region. Prior work has demonstrated autonomous healing in coatings containing electrospun fibers, but full characterization of the electrospun fiber microstructure and healing performance of the coating is lacking. In this study, we utilize electrospun fibers containing liquid healing agents to achieve a crosslinking reaction of poly(dimethylsiloxane) (PDMS) to a crosslinking agent poly(diethoxysiloxane) (PDES), initiated by the catalyst dibutyltindilaurate (DBTL), to fill a damaged region and reseal the metal substrate. Fiber morphology is characterized using scanning electron microscopy (SEM), transmission electron microscopy (TEM), and confocal fluorescence microscopy (CFM). Successful delivery of healing agents to the damage region and subsequent crosslinking reaction is observed using SEM and chemically using infrared spectroscopy. The performance of the healed coating is evaluated electrochemically using linear polarization, where the coatings were subjected to a corrosive environment. The self-healing electrospun coating exhibits lower corrosion current than in control cases, resulting in an $88 \%$ corrosion inhibition efficiency.
\end{abstract}




\section{Introduction}

One of the most common methods to protect metals from corrosion is the application of a protective polymer coating. Once a coating is damaged and the underlying material exposed, the protective ability is compromised and corrosion initiates. Self-healing strategies have been explored to restore protection to damaged coatings[1-8]. Autonomous repair can increase the lifetime of coatings, thereby reducing the economic and environmental impact of corrosion.

Two common self-healing strategies for coating materials are intrinsic and capsule-based systems[5]. Intrinsic systems achieve healing through reversible hydrogen bonding[6], metalligand bonding[9], or covalent bonding[8]. Kotteritzsch et al. demonstrated that coatings made from polymers with complementary Diels-Alder chemistry within the same polymer chain reversibly heal upon thermal treatment[8]. Although the reversible bonding intrinsic systems can provide multiple healing cycles, external intervention (such as thermal treatment of the damaged material) is typically needed to induce healing. Capsule-based healing systems have been implemented for a wide range of materials and applications[5]. In this strategy, healing agents are encapsulated and dispersed in a matrix. Upon damage the capsules rupture, releasing their contents into the damaged region. Common capsule-based sequestration approaches include capsules that react to 1) a catalyst phase that is dispersed in the matrix, 2) a second capsule with a complementary chemistry, 3) a latent functionality in the matrix, and 4) reactive agents that are phase separated in the matrix[5]. Cho et al. demonstrated self-healing of a polymeric coating containing microencapsulated tin catalyst and phase separated poly(dimethylsiloxane)[1]. Upon mechanical damage, the capsules released the catalyst into the damage zone, which reacted with the phase separated polymer thereby resealing the coating and effectively protecting the underlying steel substrate from corrosion. 
Fibers are another promising strategy for storing healing agents in a material[3,10-13]. One versatile method for producing fibers is electrospinning. Electrospinning (ES) produces fibers by applying a high voltage to a solution droplet that is delivered through a metallic nozzle. When sufficient surface charge builds up on the droplet, the solution surface is stretched and a Taylor cone is formed[14]. As electrical force overcomes the surface energy, fluid jets are ejected from the solution surface and accelerate through the electric field. As the jet travels towards the grounded electrode, the volatile solvents evaporate resulting in solid fibers at the collector. ES typically produces nonwoven fibers in the diameter range of $10 \mathrm{~nm}$ to 10 $\mu \mathrm{m}[15,16]$. Moreover, a wide range of materials can be processed using this method, including polymers, ceramics, and inorganics[15-23]. The versatility of ES allows for tailoring to specific applications by strategic selection of starting materials.

In coaxial ES, two immiscible liquids are pumped through inner and outer coaxial needles to form a core-shell fiber, as shown in Figure 1. Using this modification to ES the core could be either liquid or solid[24-26]. For self-healing applications, the inner liquid is the healing agent and the outer liquid forms the encapsulating polymer shell wall. Coaxially spun materials have been utilized a variety of applications, such as biomedical applications for tissue engineering and drug delivery[27-29]. The use of ES for encapsulating healing agents has only recently been explored. Mitchell and Keller showed that fibers containing an epoxy healing agent could be synthesized but no demonstration of healing ability was discussed[10]. Park and Braun reported a self-healing coating system with a two-part polysiloxane based healing system contained in electrospun fibers[3]. Upon damage, the healing agents in the fibers wicked into the crack plane. Although this coating system successfully reduced corrosion in a damaged region, the degree of mixing and crosslinking reaction between the two healing agents was not 
evaluated. Without confirmation that the healing agents successfully reacted, the coatings may have only unreacted (liquid) healing agents in the damage region, which acts only as a temporary physical barrier between the metal substrate and the environment. Electrospinning has also been used to develop flexible transparent self-healing coatings for optical devices [30]. In addition to coatings, electrospun fibers have also been used to add healing functionality into composites[11,31-35]. Lee et al. reported an electrospun fiber system containing two-part polysiloxane healing chemistry that recovered mechanical properties (Young's modulus and adhesion) after several cycles of mechanical testing[34,35]. Wu et al. reported a system where electrospun fibers containing dicyclopentadience (DCPD) were incorporated between carbon fiber plies and processed to make a composite that could recover some mechanical properties after damage, due to the reaction of DCPD with the Grubb's catalyst in the matrix [11].

In this work, we develop a self-healing coating material with electrospun core-shell fibers to protect steel. We focus our efforts on characterizing the morphology of the electrospun fibers and the healing performance of the coating through the use of several microscopy techniques, infrared spectroscopy, and electrochemical characterization. In our coatings, the liquid healing agents are contained in two types of fibers, type A and B. The core material of type A fibers contain a stoichiometric mixture of poly(dimethylsiloxane) and crosslinker poly(diethoxysiloxane) (PDES), while the core material of type B fibers contain the crosslinking catalyst dibutyltindilaurate (DBTL). When the fibers rupture, the healing agents mix and initiate crosslinking of polysiloxanes. This healing system is chosen due to prior success in repairing bulk materials and coatings when microencapsulated[7,36,37]. Several microscopy methods are employed to characterize these fibers and confirm successful encapsulation of healing agents. Furthermore, we also explore how experimental parameters of ES affect fiber morphology and 
adjust accordingly to eliminate the bead-on-string morphology and produce smooth fibers. The fibers are embedded in a polymer matrix to produce a coating and subsequently damaged to elicit an autonomic healing response. Healing performance is evaluated visually (SEM), chemically (FTIR), and electrochemically (linear polarization).

\section{Experimental}

\subsection{Materials}

Poly(vinyl alcohol) (PVA, 87-89\% hydrolyzed, average $\mathrm{M}_{\mathrm{w}}=85,000-124,000 \mathrm{~g} / \mathrm{mol}$, Sigma-Aldrich), silanol terminated poly(dimethylsiloxane) (PDMS, DMS-S21, Gelest), poly(diethoxysiloxane) (PDES, PSI-021, Gelest), dibutyltindilaurate (DBTL, Gelest), sodium chloride (Sigma-Aldrich), acetone (Sigma-Aldrich), sudan blue (Sigma-Aldrich), coumarin 6 (Sigma-Aldrich), and silicone conformal coating (KO805A, Miller-Stephenson) were used without further purification. Deionized water $\left(\mathrm{DI} \mathrm{H}_{2} \mathrm{O}\right)$ was prepared using a Milli-Q Biocel Millipore purification system and had a resistivity of at least $18 \mathrm{M} \Omega / \mathrm{cm}$. Purchased hot rolled carbon steel sheets ( $4.76 \mathrm{~mm}$ thick) were sandblasted (180 grit), rinsed in acetone, and sprayed with compressed air before use.

\subsection{Coaxial electrospinning}

\subsubsection{Preparation of electrospinning solutions}

Two types of core-shell electrospun fibers were fabricated: type A (PDMS) and type B (DBTL catalyst). The core of type A (PDMS) fibers contained a solution of PDMS (main healing agent) and PDES (crosslinking agent). This solution was made by mixing $3.2 \mathrm{~g}$ of PDMS 
and $2.9 \mathrm{~g}$ of PDES, and was allowed to stir overnight before use. The core material of type B (DBTL catalyst) fibers contained the crosslinking catalyst DBTL and was used as received. The shell of both types of fibers was PVA, prepared as an aqueous solution of $20 \%$ by wt. PVA powder in $\mathrm{DI} \mathrm{H}_{2} \mathrm{O}$ and stirred for 24 hours before use.

\subsubsection{Electrospinning conditions}

Our custom-built electrospinning setup is shown schematically in Fig. 1. A portion of the PVA shell solution was drawn into a $5 \mathrm{~mL}$ syringe and affixed within a syringe pump (KDScientific, Model 780101). The syringe was then connected to a sealed stainless steel coaxial needle (Linari Biomedical, Model COAX-2DISP, inner (core) needle: outer diameter $(\mathrm{OD}) /$ inner diameter $(\mathrm{ID})=0.83 \mathrm{~mm} / 0.51 \mathrm{~mm}$, outer $($ shell $)$ needle: $\mathrm{OD} / \mathrm{ID}=1.83 \mathrm{~mm} / 1.37 \mathrm{~mm}$ ) via polypropylene luer lock couplings and chemical-resistant poly(vinyl carbonate) (PVC) Tygon tubing $(\mathrm{OD} / \mathrm{ID}=4.76 \mathrm{~mm} / 1.59 \mathrm{~mm})$. Another syringe was filled with either type A (PDMS) solution or type B (DBTL catalyst) liquid and attached to the coaxial needle and a second syringe pump in a similar fashion. The syringe pump flow rates were $6.0 \mu \mathrm{L} / \mathrm{min}$ (shell) and $3.0 \mu \mathrm{L} / \mathrm{min}$ (core). A high voltage power supply (Spellman, Bertan Series 230) was connected to the metal needle with an electrode. For a vertical electrospinning orientation, a 10 x $10 \mathrm{~cm}$ steel collector plate was mounted $14 \mathrm{~cm}$ above the needle and connected to the ground electrode on the power supply. For each coating sample, a new 5 x $5 \mathrm{~cm}$ hot-rolled, sandblasted (180 grit) carbon steel coupon was attached to the collector using conductive carbon tape. The voltages used for electrospinning were $12 \mathrm{kV}$ (type A) and $15 \mathrm{kV}$ (type B). Additionally, the effect of varying the shell solution flow rate was also studied in order to optimize core-shell fiber morphology. The 
solution flow rate for the shell material was varied from $4 \mu \mathrm{L} / \mathrm{min}$ to $6 \mu \mathrm{L} / \mathrm{min}$, while all other variables remained constant.

\subsection{Characterization of core-shell fibers}

The core-shell fibers were characterized using several microscopy techniques including scanning electron microscopy (SEM), transmission electron microscopy (TEM), and confocal fluorescence microscopy (CFM). The surface morphology of the core-shell fibers was visualized using SEM (FEI/Philips XL30 ESEM-FEG). The samples were prepared by affixing the ES fibers deposited on the steel sheet to a metal stub using conductive carbon tape and then sputtercoated with a thin layer of Au/Pd before SEM analysis. The samples were imaged at an accelerating voltage of 3-5 kV. The core-shell structure was observed via TEM (JEOL 2100 Cryo TEM) and CFM(Leica TCS SP2 RBB). For TEM, the fibers were electrospun onto a steel parallel plate collector that resulted in fibers suspended in a $0.5 \mathrm{~cm}$ gap in the collector. A carbon coated copper TEM grid was then placed in the gap to gently lift off fibers from the collector onto the grid. The samples were imaged via TEM without further preparation. For CFM, the solutions for the core material were fluorescently dyed before electrospinning. To prepare the dyed core solutions, the dye powder (Sudan blue for type A (PDMS), coumarin 6 for type B (DBTL catalyst)) was mixed with the core material and allowed to stir overnight to produce a $0.01 \%$ dyed core solution. The dyed fiber samples were analyzed without further preparation after the fibers were deposited onto the steel. The samples were illuminated with a laser $(488 \mathrm{~nm})$ and fluorescence micrographs were collected at 637-678 nm for type A (PDMS) and 501-546 nm for type B (DBTL catalyst). 


\subsection{Coating fabrication}

The self-healing coatings were produced by electrospinning alternate layers of fiber type A (PDMS) and type B (DBTL catalyst), which were then permanently fixed to steel substrate using a silicone binder matrix. A schematic of the coating fabrication is shown in Fig. 2. For each sample, a 5 x 5 cm hot-rolled steel sheet was sandblasted (180 grit), sprayed with air, rinsed with acetone, and sprayed with compressed air. The electrospun fibers were deposited directly onto the steel coupon, alternating layer by layer of each fiber type. The first layer (type B, DBTL catalyst) was deposited for $1 \mathrm{hr}$ and then dried in a desiccator for $1 \mathrm{hr}$. The process was repeated with type A (PDMS) fibers, until 4 layers were deposited to obtain a coating where the order of electrospinning was type B, A, B, and A. Each fiber layer was approximately the same thickness $(\sim 5 \mu \mathrm{m})$, since the electrospinning deposition time controls thickness and was kept constant. A silicone binder was spun cast (500 rpm for $60 \mathrm{sec})$ onto the fibers and cured at room temperature for 24 hours to produce the final coating material (thickness $\sim 20 \mu \mathrm{m}$ ). For control experiments, coatings containing no fibers (silicone binder only), coating with type A (PDMS) fibers (electrospinning time $=4 \mathrm{hr}$ ) and silicone binder, and coating with type B (DBTL catalyst) fibers (electrospinning time $=4 \mathrm{hr}$ ) and silicone binder were produced, and each control type had a final coating thickness of about $20 \mu \mathrm{m}$.

For healing experiments, the coating was damaged using a corrocutter (Erichsen Model 639) with a razor blade tip to produce a $2.5 \mathrm{~cm}$ length linear scribe. The coating was allowed to heal at room temperature for $24 \mathrm{hr}$ before further characterization. These control coatings were similarly damaged with a corrocutter and characterized $24 \mathrm{hr}$ after scribing. 


\subsection{Characterization of coating and corrosion inhibition}

\subsubsection{Microscopy of damaged region}

Healing performance of self-healing and control coatings was assessed by microscopy (SEM), chemical analysis (FTIR), and electrochemical analysis (potentiostat). For SEM, all scribed coatings were mounted onto a metal stub using conductive carbon tape and sputtercoated for $70 \mathrm{~s}$ to obtain a thin layer of $\mathrm{Au} / \mathrm{Pd}$ before analysis. Images of the coating surface were taken to visualize healing agent release into the scribed region. The healed coating was submerged in PET ether (solvent for unreacted PDMS) for 15 min, allowed to dry, and then analyzed under SEM to visually confirm the crosslinking of the healed region. A control case was performed by comparing the healed coating to a type A (PDMS) fibers only coating.

\subsubsection{Chemical analysis of coating}

Chemical analysis of the coatings was performed using Fourier transform infrared spectroscopy (FTIR, Perkin Elmer Spotlight 400 with a liquid nitrogen cooled mercury cadmium telluride (MCT) linear array detector). Pixel size at the sample plane was $6.25 \mu \mathrm{m}$ and the spectral range collected was $4000-750 \mathrm{~cm}^{-1}$. Spectra were acquired at a $8 \mathrm{~cm}^{-1}$ resolution using an interferometer speed of $1 \mathrm{~cm} / \mathrm{s}$ with a single scan. For each image, 64 scans were averaged per pixel and the scan area was variable. The coating samples used for FTIR analysis were electrospun fibers on steel without a silicone binder to ensure that contributions to the FTIR data were only from the fibers. Healed regions and undamaged regions on the coating were examined. FTIR spectra of the undamaged region was acquired from a region at least $10 \mu \mathrm{m}$ away from the scribed area. The fiber samples were analyzed without further preparation. Neat solutions of the core materials (PDMS and DBTL) and a mixture of the two neat solutions (allowed to react for at least $24 \mathrm{hr}$ ) were also analyzed for comparison. 


\subsubsection{Electrochemical analysis of coating}

For electrochemical analysis, the coatings were characterized using a linear polarization sweep (BioLogic, Model VSP). The three-electrode system consisted of the coating on steel for the working electrode, a Pt counter electrode, and a $\mathrm{Ag} / \mathrm{AgCl}$ reference electrode. Each specimen was exposed to a freshly prepared aqueous salt solution $(5 \% \mathrm{NaCl}, 30 \mathrm{~mL}$ total volume) on the coated side and immediately connected to the potentiostat. The samples were allowed to equilibrate for $20 \mathrm{~min}$ and the voltage was recorded every $0.100 \mathrm{~s}$ during this resting period. The final voltage at the end of the resting period was used as the open circuit voltage, $E_{\mathrm{oc}}$. The potential of the working electrode was from the range of $-0.100 \mathrm{~V}$ vs. $E_{\mathrm{oc}}$ to $0.100 \mathrm{~V}$ vs. $E_{\mathrm{oc}}$ at a rate of $0.200 \mathrm{mV} / \mathrm{s}$. The experimental results were plotted as the logarithm of the current vs. the voltage of the working electrode (log $I$ vs. $E_{\text {we }}$ ) and then processed using a commercial software (Bio-Logic EC-Lab Software version 10.34) to obtain a Tafel fit. The Tafel plot was calculated by minimizing the chi-squared $\left(\chi^{2}\right)$ distribution value. The corrosion current $\left(I_{\text {corr }}\right)$ was determined by the intersection of the slopes of the Tafel plot and averaged over 5 samples (a representative curve is shown in Fig. 9). Corrosion inhibition efficiency (IE\%) was defined by Eq. 1, where $I_{\text {corr }}^{\text {control }}$ is the average corrosion current for control coatings with no fibers and $I_{\text {corr }}^{\text {sample }}$ is the corrosion current for a coating with fibers:

$$
I E \%=\frac{I_{\text {corr }}^{\text {cont }}-I_{\text {corr }}^{\text {sample }}}{I_{\text {corr }}^{\text {control }}} \times 100 \%
$$

\subsubsection{Visual analysis of coatings after exposure to corrosion}

The coatings were observed for visual signs of corrosion after prolonged immersion in the subjected corrosive aqueous salt solution for electrochemical evaluation. When linear polarization measurements were completed, the coatings were removed from the test chamber 
and allowed to dry. The samples were stored for 4 months and images of the coatings were taken using a digital camera.

\section{Results and Discussion}

\subsection{Fiber characterization}

\subsubsection{Surface and core-shell fiber morphology}

Smooth fibers with uniform diameters containing encapsulated healing agents were produced from electrospinning. SEM micrographs (Figs 3a, 3d) show that fibers are randomly deposited on to the steel substrate and have an average diameter of $300 \mathrm{~nm}$ (type A, PDMS) and $400 \mathrm{~nm}$ (type B, DBTL catalyst). The full distribution of fiber diameters is included in Supporting Information. TEM and CFM were performed to verify the core-shell structure. TEM of individual fibers reveals distinctive core-shell morphology (Figs. 3b, 3e). Based on the inner core diameter in these images, we estimate the volume fraction healing agent is approximately $41 \%$ for fiber type A and $12 \%$ for fiber type B. Hence, we estimate the ratio of A:B available to the crack region to be 7:2. CFM images (Figs. 3c, 3f) confirmed uniform distribution of core material throughout the fibers and that most fibers contained core material.

\subsubsection{Control of fiber morphology}

Electrospun core-shell fibers reported in the literature are produced in different shapes, such as bead-on-string and straight uniform fibers[15,16]. Here, the fiber morphology can be controlled by varying the solution flow rate for the shell material. As shown in Fig. 4, lower shell solution pumping rates led to the bead-on-string morphology $(4.0 \mu \mathrm{L} / \mathrm{min})$, while a higher pumping rate resulted in fibers with uniform diameters $(6.0 \mu \mathrm{L} / \mathrm{min})$. This trend is consistent with prior work that showed that the spacing between the beads and bead diameter was controlled by varying the inner solution flow rate[38]. Fiber morphology can be tailored to 
specific applications, where a certain morphology type may be favored. For self-healing coatings using coaxially electrospun fibers, prior studies[3,10] only utilized the bead-on-string morphology, where the healing agent core material was concentrated in discrete regions along a fiber. We anticipate that bead-free fibers will result in more uniform distribution of healing agents and improved healing performance.

\subsection{Coating characterization}

\subsubsection{Visual analysis of healed coatings}

Coatings containing self-healing fibers and control coatings with no fibers, i.e. silicone binder coating only, were damaged and compared under SEM to inspect for evidence of healing. As expected, images of control coatings that did not contain fibers revealed only exposed steel substrate in the scribed region (Fig. 5a). In contrast, images of self-healing coatings (Fig. 5b) showed polymerized material in the damage zones, indicating that the liquid core materials contained in the electrospun fibers were released into the damaged region. Although the healed material did not completely fill the entire depth of the damage region, it provided some level of protection as long as the exposed metal surface was covered. We confirmed that the material in the damaged region of the self-healing coating was crosslinked PDMS, by immersing both selfhealing samples and control samples with type A (PDMS) fibers only were rinsed in an organic solvent for PDMS (PET ether) to remove any unreacted healing agents (PDMS and DBTL). PDMS and PDES will not react without the presence of the catalyst (DBTL) [7,37]. As expected, bare steel was observed for the damaged type A (PDMS) coating after solvent treatment, (Fig. 5c and 5e). For comparison, a bare steel sample was also scribed and imaged (see Supporting 
Information). In contrast, polymerized material remained present in the damaged region of the self-healing coating (Fig. 5d).

\subsubsection{Chemical analysis of healed coatings}

FTIR analysis was used to characterize chemical changes that occurred after release of the healing agents in the crack plane. Fig. 6 summarizes the chemical analysis of different areas of the scribed sample along with the authentic components in the coating for comparison. FTIR samples of the healed and undamaged region were of the electrospun fibers only and did not contain the silicone binder to simplify data interpretation.

The FTIR spectra in the undamaged region (Fig 6a) has the characteristic bands corresponding to PDMS and crosslinker PDES from Fig. 6b, as well as some contribution from the PVA shell wall (-OH stretch at $\left.3300-3400 \mathrm{~cm}^{-1}\right)$. The presence of PDMS, PDES, and PVA peaks is expected since the top fiber layer is composed of type A (PDMS) fibers. The spectra for type A core material solution containing PDMS and crosslinker PDES is shown in Fig. 6b. The characteristic C-H stretching of $\mathrm{CH}_{3}$ at $2964 \mathrm{~cm}^{-1}, \mathrm{CH}_{3}$ symmetric deformation of $\mathrm{Si}-\mathrm{CH}_{3}$ at $1256 \mathrm{~cm}^{-1}$, Si-O-Si stretching vibration at $1080 \mathrm{~cm}^{-1}$ and $1012 \mathrm{~cm}^{-1}$, and Si-C stretching and $\mathrm{CH}_{3}$ rocking at $862 \mathrm{~cm}^{-1}$ and $792 \mathrm{~cm}^{-1}$ are observed. The peak values are in agreement with the literature[39,40].

For comparison, the PDMS with PDES solution (Fig. 6b) and DBTL catalyst (Fig. 6c) were simply mixed in a 1:1 ratio and allowed to react for $24 \mathrm{hr}$. The FTIR spectrum for the mixed solution showed contributions from both healing agents (Fig 6e). This spectrum can be

separated into three regions and attributed to the corresponding chemical agent: $2800-2950 \mathrm{~cm}^{-1}$ (alkyl stretch from DBTL and PDMS), 1300-1750 $\mathrm{cm}^{-1}$ (DBTL), and 790-1260 $\mathrm{cm}^{-1}$ (mostly PDMS with some small contribution from DBTL). The FTIR spectrum from the healed region 
(Fig 6d) was observed to be very similar to the mixture of PDMS and DBTL (Fig. 6e), indicating that both types of fibers were released into the damaged region and mixed.

To determine the difference in the FTIR spectrum between crosslinked and uncrosslinked polymer, we collected FTIR spectra from PDMS specimens of different crosslink densities and used to compare to the spectrum of the healed coating (see Supporting Information, Fig SI4). A drop of PDMS and PDES was placed next to a droplet of DBTL catalyst, allowed to react for 24 hr, and then spatially analyzed with FTIR. Crosslinking density was determined spatially (PDMS in areas near DBTL catalyst would have higher crosslinking) and confirmed by changes in spectral peaks. FTIR spectroscopy showed that increasing crosslink density resulted in an increased wavenumber shift for the Si-O-Si peak around $1120-1010 \mathrm{~cm}^{-1}$ and Si-C stretching and $\mathrm{CH}_{3}$ rocking around 862 and $792 \mathrm{~cm}^{-1}$. The upshift in these and related peaks have been used to track PDMS crosslinking in other studies[41]. The presence of a broad range of peaks

from $1012-1200 \mathrm{~cm}^{-1}$ indicates that the healed region contains several chemical species that vary in crosslinking density.

\subsubsection{Electrochemical analysis and corrosion inhibition}

The self-healed and control coatings were inspected after exposure to corrosive aqueous salt solution during electrochemical testing for visual signs of corrosion. Optical images of the coatings 4 months after exposure to a corrosive environment are shown in Fig. 7. Before electrochemical analysis, the scribed coatings had no visual signs of corrosion. After exposure to the corrosive environment, the three control cases containing type A (PDMS) fibers only, type B (DBTL catalyst) fibers only, and no fibers all have significant onset of corrosion damage and undercutting. In contrast, the healed coating showed minimal signs corrosion restricted to only the scribed region. 
The protective performance of the self-healing and control coatings were also evaluated by electrochemical analysis. Linear polarization measurements of the healed sample and each of the control cases (no fibers, type A (PDMS), type B (DBTL catalyst)) are shown in Fig. 8. The corrosion current increased with applied potential. The corrosion current for healed coatings was significantly lower than the control coatings, indicating effective healing behavior and reduction of corrosion. To quantify corrosion inhibition, we determined the corrosion current $\left(I_{\text {corr }}\right)$ by extrapolation of the cathodic and anodic Tafel lines for each sample. A representative curve for a control case (no fibers) with Tafel extrapolation is shown in Supporting Information. The corrosion current and corrosion inhibition efficiency (IE\%) are reported for the healed coating and the control cases (Fig. 9).

The corrosion current for the healed coating was $0.17 \pm 0.10 \mu \mathrm{A}$, an order of magnitude less than the control cases. Using Eq. (1), a corrosion inhibition efficiency of $88 \%$ corrosion was calculated for the self-healing coating. The corrosion current values for the control cases were $1.46 \pm 0.32 \mu \mathrm{A}$ (no fibers), $1.86 \pm 0.22 \mu \mathrm{A}$ (type A fibers only), and $1.24 \pm 0.24 \mu \mathrm{A}$ (type B fibers only). The higher rate of corrosion observed in the type A (PDMS) coatings was attributed to the hydrolysis of uncrosslinked PDMS core material, which could lead to partial disintegration of coating material[42]. Electrospun PVA nanofibers also have been shown to reduce corrosion for aluminum[43], which could explain the slightly lower corrosion rate of the type B (DBTL catalyst) coatings as compared to the coatings with no fibers. Lastly, the corrosion current for an undamaged coating was $\mathrm{I}_{\text {corr }}=0.002 \mu \mathrm{A}$, indicating that the undamaged coating is an effective barrier against corrosion and that a coating with a $100 \%$ healing efficiency should approach this value. 


\section{Conclusions}

The self-healing performance of a polymer coating containing electrospun core-shell fibers of PDMS based healing agents were characterized via SEM, TEM, and confocal fluorescence microscopy. The beading morphology was controlled by optimization of solution flow rate during electrospinning. Damage initiated by a scribe caused a catalyst-initiated crosslinking reaction of poly(dimethylsiloxane) (PDMS) and poly(diethoxysiloxane) (PDES). Crosslinking of the released core materials was verified using FTIR analysis, as evidenced by the broad range of peaks from the Si-O-Si bond around 1012-1200 $\mathrm{cm}^{-1}$ shifting 10 to $50 \mathrm{~cm}^{-1}$ higher. SEM images revealed new polymer in the healed region even after a solvent treatment. Finally, the effectiveness of the healed coating towards preventing corrosion was demonstrated using linear polarization experiments, where the healed coating exhibited $88 \%$ corrosion inhibition efficiency.

\section{Acknowledgements}

The authors would like to acknowledge the funding provided by the BP International Centre for Advanced Materials (BP-ICAM) in collaboration with the University of Illinois at Urbana-Champaign (UIUC). T. Doan is supported by the National Defense Science and Engineering Graduate (NDSEG) Fellowship, which is under Government support from contract FA9550-11-C-0028 and awarded by the Department of Defense, Air Force Office of Scientific Research, 32 CFR 168a. T. Doan obtained and analyzed all data unless otherwise mentioned. L.S. Leslie and R. Bhargava obtained FTIR data, S.Y. Kim helped in obtaining the confocal fluorescence images for Fig. 4d-f, and N.R. Sottos and S.R. White provided advising. The authors would like to thank the Autonomous Materials Systems group at UIUC for their support. 


\section{References}

[1] S.H. Cho, S.R. White, P. V. Braun, Self-Healing Polymer Coatings, Adv. Mater. 21 (2009) 645-649. doi:10.1002/adma.200802008.

[2] G. Scheltjens, M.M. Diaz, J. Brancart, G. Van Assche, B. Van Mele, A self-healing polymer network based on reversible covalent bonding, React. Funct. Polym. 73 (2013) 413-420. doi:10.1016/j.reactfunctpolym.2012.06.017.

[3] J.-H. Park, P. V Braun, Coaxial electrospinning of self-healing coatings., Adv. Mater. 22 (2010) 496-9. doi:10.1002/adma.200902465.

[4] K.S. Toohey, N.R. Sottos, S.R. White, Characterization of Microvascular-Based Selfhealing Coatings, Exp. Mech. 49 (2008) 707-717. doi:10.1007/s11340-008-9176-7.

[5] B.J. Blaiszik, S.L.B. Kramer, S.C. Olugebefola, J.S. Moore, N.R. Sottos, S.R. White, SelfHealing Polymers and Composites, Annu. Rev. Mater. Res. 40 (2010) 179-211. doi:10.1146/annurev-matsci-070909-104532.

[6] F. Deflorian, S. Rossi, E. Scrinzi, Self-healing supramolecular polyurethane coatings: preliminary study of the corrosion protective properties, Corros. Eng. Sci. Technol. 48 (2013) 147-154. doi:10.1179/1743278212Y.0000000062.

[7] S.H. Cho, H.M. Andersson, S.R. White, N.R. Sottos, P.V. Braun, PolydimethylsiloxaneBased Self-Healing Materials, Adv. Mater. 18 (2006) 997-1000.

doi:10.1002/adma.200501814.

[8] J. Kötteritzsch, S. Stumpf, S. Hoeppener, J. Vitz, M.D. Hager, U.S. Schubert, Onecomponent intrinsic self-healing coatings based on reversible crosslinking by diels-alder cycloadditions, Macromol. Chem. Phys. 214 (2013) 1636-1649.

doi:10.1002/macp.201200712.

[9] S. Bode, L. Zedler, F.H. Schacher, B. Dietzek, M. Schmitt, J. Popp, M.D. Hager, U.S. Schubert, Self-healing polymer coatings based on crosslinked metallosupramolecular copolymers, Adv. Mater. 25 (2013) 1634-1638. doi:10.1002/adma.201203865.

[10] T.J. Mitchell, M.W. Keller, Coaxial electrospun encapsulation of epoxy for use in selfhealing materials, Polym. Int. 62 (2013) 860-866. doi:10.1002/pi.4397.

[11] X.F. Wu, A. Rahman, Z. Zhou, D.D. Pelot, S. Sinha-Ray, B. Chen, S. Payne, A.L. Yarin, Electrospinning core-shell nanofibers for interfacial toughening and self-healing of carbon-fiber/epoxy composites, J. Appl. Polym. Sci. 129 (2013) 1383-1393. doi:10.1002/app.38838.

[12] M.W. Lee, S.S. Yoon, A.L. Yarin, Solution-Blown Core-Shell Self-Healing Nano- and Microfibers, ACS Appl. Mater. Interfaces. 8 (2016) 4955-4962. doi:10.1021/acsami.5b12358.

[13] M.W. Lee, S. Sett, S.S. Yoon, A.L. Yarin, Fatigue of Self-Healing Nanofiber-based Composites: Static Test and Subcritical Crack Propagation, ACS Appl. Mater. Interfaces. 8 (2016) 18462-18470. doi:10.1021/acsami.6b05390. 
[14] G. Taylor, Electrically Driven Jets, Proc. Roy. Soc. Lond. A. 313 (1969) 453-475.

[15] A. Greiner, J.H. Wendorff, Electrospinning: a fascinating method for the preparation of ultrathin fibers., Angew. Chemie. 46 (2007) 5670-703. doi:10.1002/anie.200604646.

[16] D. Li, Y. Xia, Electrospinning of Nanofibers: Reinventing the Wheel?, Adv. Mater. 16 (2004) 1151-1170. doi:10.1002/adma.200400719.

[17] T.J. Boyle, T.Q. Doan, L.A.M. Steele, C. Apblett, S.M. Hoppe, K. Hawthorne, R.M. Kalinich, W.M. Sigmund, Tin(II) amide/alkoxide coordination compounds for production of Sn-based nanowires for lithium ion battery anode materials., Dalt. Trans. 41 (2012) 9349-64. doi:10.1039/c2dt30772c.

[18] D. Li, Y. Wang, Y. Xia, V. Uni, Electrospinning of Polymeric and Ceramic Nanofibers as Uniaxially Aligned Arrays, Nano Lett. 3 (2003) 1167-1171.

[19] M.J. Laudenslager, R.H. Scheffler, W.M. Sigmund, Electrospun materials for energy harvesting, conversion, and storage: A review, Pure Appl. Chem. 82 (2010) 2137-2156. doi:10.1351/PAC-CON-09-11-49.

[20] P. Muthiah, S.H. Hsu, W. Sigmund, Coaxially electrospun PVDF-teflon AF and teflon AF-PVDF core-sheath nanofiber mats with superhydrophobic properties, Langmuir. 26 (2010) 12483-12487. doi:10.1021/la100748g.

[21] C. Gualandi, A. Zucchelli, M.F. Osorio, J. Belcari, M.L. Focarete, Nanovascularization of Polymer Matrix: Generation of Nanochannels and Nanotubes by Sacrificial Electrospun fibers, (2013).

[22] H. Saghafi, A. Zucchelli, R. Palazzetti, G. Minak, The effect of interleaved composite nanofibrous mats on delamination behavior of polymeric composite materials, Compos. Struct. 109 (2014) 41-47. doi:10.1016/j.compstruct.2013.10.039.

[23] R. Qing, W. Sigmund, Morphological and crystallite size impact on electrochemical performance of electrospun rutile and rutile/multiwall carbon nanotube nanofibers for lithium ion batteries, Ceram. Int. 40 (2014) 5665-5669.

doi:10.1016/j.ceramint.2013.11.003.

[24] A.K. Moghe, B.S. Gupta, Coaxial Electrospinning for Nanofiber Structures: Preparation and Applications, Polym. Rev. 48 (2008) 353-377. doi:10.1080/15583720802022257.

[25] F. Li, Y. Zhao, Y. Song, Core-Shell Nanofibers : Nano Channel and Capsule by Coaxial Electrospinning, in: Nanofibers, 2010: pp. 419-438.

[26] Z. Sun, E. Zussman, A.L. Yarin, J.H. Wendorff, A. Greiner, Compound Core-Shell Polymer Nanofibers by Co-Electrospinning, Adv. Mater. 15 (2003) 1929-1932. doi:10.1002/adma.200305136.

[27] C. Wang, K.W. Yan, Y.-D. Lin, P.C.H. Hsieh, Biodegradable Core/Shell Fibers by Coaxial Electrospinning: Processing, Fiber Characterization, and Its Application in Sustained Drug Release, Macromolecules. 43 (2010) 6389-6397. doi:10.1021/ma100423x.

[28] H. Jiang, Y. Hu, Y. Li, P. Zhao, K. Zhu, W. Chen, A facile technique to prepare biodegradable coaxial electrospun nanofibers for controlled release of bioactive agents., J. Control. Release. 108 (2005) 237-43. doi:10.1016/j.jconrel.2005.08.006. 
[29] L. Buttafoco, N.G. Kolkman, P. Engbers-Buijtenhuijs, a a Poot, P.J. Dijkstra, I. Vermes, J. Feijen, Electrospinning of collagen and elastin for tissue engineering applications., Biomaterials. 27 (2006) 724-34. doi:10.1016/j.biomaterials.2005.06.024.

[30] S. An, M. Liou, K.Y. Song, H.S. Jo, M.W. Lee, S.S. Al-Deyab, A.L. Yarin, S.S. Yoon, Highly flexible transparent self-healing composite based on electrospun core-shell nanofibers produced by coaxial electrospinning for anti-corrosion and electrical insulation., Nanoscale. 7 (2015) 17778-85. doi:10.1039/c5nr04551g.

[31] V. Vahedi, P. Pasbakhsh, C.S. Piao, C.E. Seng, A facile method for preparation of selfhealing epoxy composites: using electrospun nanofibers as microchannels, J. Mater. Chem. A. 3 (2015) 16005-16012. doi:10.1039/C5TA02294K.

[32] M.W. Lee, S. An, C. Lee, M. Liou, A.L. Yarin, S.S. Yoon, Hybrid Self-Healing Matrix Using Core - Shell Nano fibers and Capsuleless Microdroplets, (2014). doi:10.1021/am5020293.

[33] S. Sinha-Ray, D.D. Pelot, Z.P. Zhou, A. Rahman, X.-F. Wu, a. L. Yarin, Encapsulation of self-healing materials by coelectrospinning, emulsion electrospinning, solution blowing and intercalation, J. Mater. Chem. 22 (2012) 9138. doi:10.1039/c2jm15696b.

[34] M.W. Lee, S. An, H.S. Jo, S.S. Yoon, A.L. Yarin, Self-Healing Nanofiber-Reinforced Polymer Composites. 1. Tensile Testing and Recovery of Mechanical Properties, ACS Appl. Mater. Interfaces. 7 (2015) 19546-19554. doi:10.1021/acsami.5b05998.

[35] M.W. Lee, S. An, H.S. Jo, S.S. Yoon, A.L. Yarin, Self-healing Nanofiber-Reinforced Polymer Composites. 2. Delamination/Debonding and Adhesive and Cohesive Properties, ACS Appl. Mater. Interfaces. 7 (2015) 19555-19561. doi:10.1021/acsami.5b03470.

[36] B.A. Beiermann, M.W. Keller, N.R. Sottos, Self-healing flexible laminates for resealing of puncture damage, Smart Mater. Struct. 18 (2009) 85001. doi:10.1088/09641726/18/8/085001.

[37] C.L. Mangun, A.C. Mader, N.R. Sottos, S.R. White, Self-healing of a high temperature cured epoxy using poly(dimethylsiloxane) chemistry, Polymer (Guildf). 51 (2010) 40634068. doi:10.1016/j.polymer.2010.06.050.

[38] J.E. Díaz, A. Barrero, M. Márquez, I.G. Loscertales, Controlled Encapsulation of Hydrophobic Liquids in Hydrophilic Polymer Nanofibers by Co-electrospinning, Adv. Funct. Mater. 16 (2006) 2110-2116. doi:10.1002/adfm.200600204.

[39] K. Berean, J.Z. Ou, M. Nour, K. Latham, C. McSweeney, D. Paull, A. Halim, S. Kentish, C.M. Doherty, A.J. Hill, K. Kalantar-zadeh, The effect of crosslinking temperature on the permeability of PDMS membranes: Evidence of extraordinary $\mathrm{CO} 2$ and $\mathrm{CH} 4$ gas permeation, Sep. Purif. Technol. 122 (2014) 96-104. doi:10.1016/j.seppur.2013.11.006.

[40] D. Cai, A. Neyer, R. Kuckuk, H.M. Heise, Raman, mid-infrared, near-infrared and ultraviolet-visible spectroscopy of PDMS silicone rubber for characterization of polymer optical waveguide materials, J. Mol. Struct. 976 (2010) 274-281.

doi:10.1016/j.molstruc.2010.03.054.

[41] A.C.C. Esteves, J. Brokken-Zijp, J. Laven, H.P. Huinink, N.J.W. Reuvers, M.P. Van, G. de With, Influence of cross-linker concentration on the cross-linking of PDMS and the network structures formed, Polymer (Guildf). 50 (2009) 3955-3966. 
doi:10.1016/j.polymer.2009.06.022.

[42] D. Graiver, K. Farminer, R. Narayan, A review of the fate and effects of silicones in the environment, J. Polym. Environ. 11 (2003) 129-136. http://link.springer.com/article/10.1023/A:1026056129717 (accessed December 16, 2014).

[43] E.M. Sherif, M. Es-saheb, A. El-zatahry, E.-R. Kenawyand, A.S. Alkaraki, Coating Electrospun Polyvinyl Alcohol and Polyvinyl Chloride Fibers As Corrosion Passivation Applications, Int. J. Electrochem. Sci. 7 (2012) 6154-6167. 


\section{List of Figures}

Figure 1 Fabrication of core-shell electrospun fibers: a) schematic of coaxial electrospinning setup, b) schematic of core-shell fibers of PVA (shell, shown in blue) and healing agent (core, shown in green), and c) SEM image of randomly deposited core-shell fibers.

Figure 2 Schematic of coating fabrication process. Samples were prepared on clean sandblasted steel by 1) electrospinning fibers directly onto steel, 2) depositing a silicone binder to affix fibers on steel, 3) scribing the coating with a corrocutter, and 4) allowing the coating to heal for $24 \mathrm{hr}$ before further characterization.

Figure 3 Evidence of core-shell fiber structure: (a) SEM, (b) TEM, and (c) fluorescence microscopy of fibers containing DBTL catalyst core with coumarin 6 fluorescent dye, (d) SEM, (e) TEM, and (f) fluorescence microscopy of fibers containing PDMS core with sudan blue fluorescent dye.

Figure 4 Effect of processing parameters on core-shell morphology. SEM and confocal fluorescence microscopy images of core-shell fibers electrospun at increasing solution pumping rate: (a, d) $4.0 \mu \mathrm{L} / \mathrm{min}$ (shell) and $3 \mu \mathrm{L} / \mathrm{min}$ (core), (b,e) 5.0 $\mu \mathrm{L} / \mathrm{min}$ (shell) and $3 \mu \mathrm{L} / \mathrm{min}$ (core), (c, f) $6.0 \mu \mathrm{L} / \mathrm{min}$ (shell) and $3 \mu \mathrm{L} / \mathrm{min}$ (core). Insets in SEM images are shown at 6500x magnification.

Figure 5 Evidence of released material in the damaged region of the coatings. SEM images of coatings (48 hours after damage) before and after solvent treatment to remove unreacted healing agents. (a) Control coating control coating without self-healing fibers and only silicone binder is shown for comparison. Before solvent treatment images of (b) self-healing coating and (c) type A (PDMS) only control coating. After solvent treatment, (d) the polymerized healing agents are retained in the scribed region and (e) unreacted PDMS is removed showing the underlying steel substrate.

Figure 6 Chemical analysis of damaged region. Summary of FTIR spectra for a) selfhealing coating in the undamaged region, b) solution of PDMS and PDES, c) neat DBTL catalyst, d) self-healing coating in the damaged region, and e) mixture of both healing agents (PDMS and DBTL). The full spectral range of the FTIR experiments are shown in (1) and the fingerprint region (2) is expanded for clarity.

Figure 7 Optical images of healed and control coatings 4 months after exposure to corrosive aqueous salt solution during electrochemical characterization. The healed coating before and after corrosion exposure is similar. However, the control cases of type A (PDMS) fibers only, type B (DBTL catalyst) fibers only, and no fibers (silicone binder only) show obvious signs of corrosion damage.

Figure 8 Electrochemical characterization of coating types. Linear polarization sweep plot of current $(I)$ vs. time for coatings containing control with silicone binder only and no fibers (black), type A (PDMS) fibers (red), and type B (DBTL catalyst) fibers (green). The healed coating (blue) has a lower corrosion current than any 
of the control cases. [Note: Curves are from one representative sample of each coating type].

Figure 9 Assessment of corrosion inhibition. Comparison of a) corrosion current $\left(\mathrm{I}_{\text {corr }}\right)$ and b) corrosion inhibition efficiency (IE\%) for each coating type: control coating with no fibers, type A (PDMS) fibers only, type B (DBTL catalyst) fiber only, and healed coating containing both type A and B fibers. [Note: vertical error bars represent the standard error from five samples tested]. 
a)

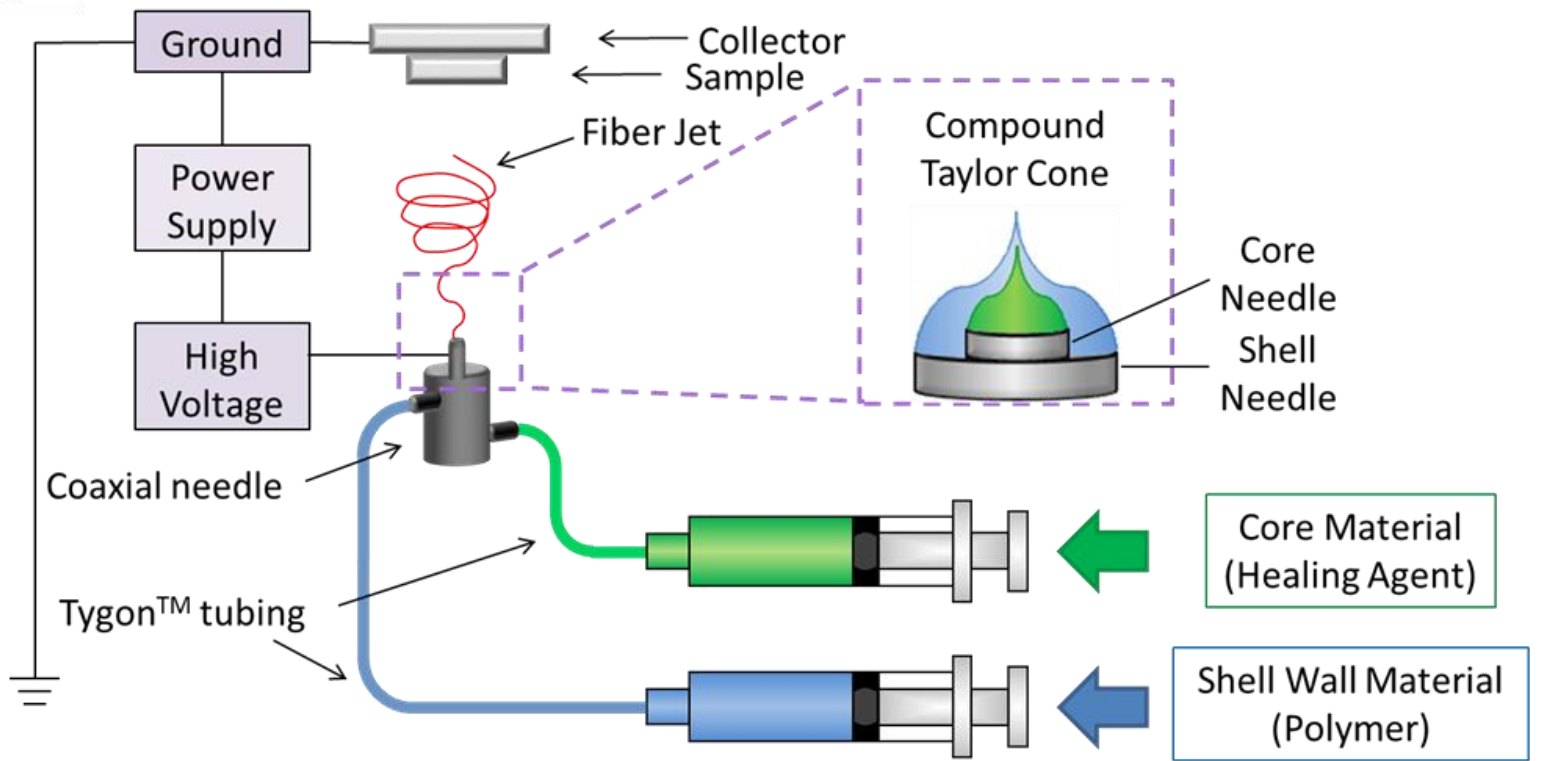

\section{b)}

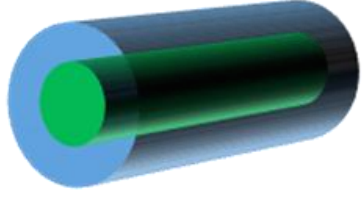

Schematic of core-shell fiber

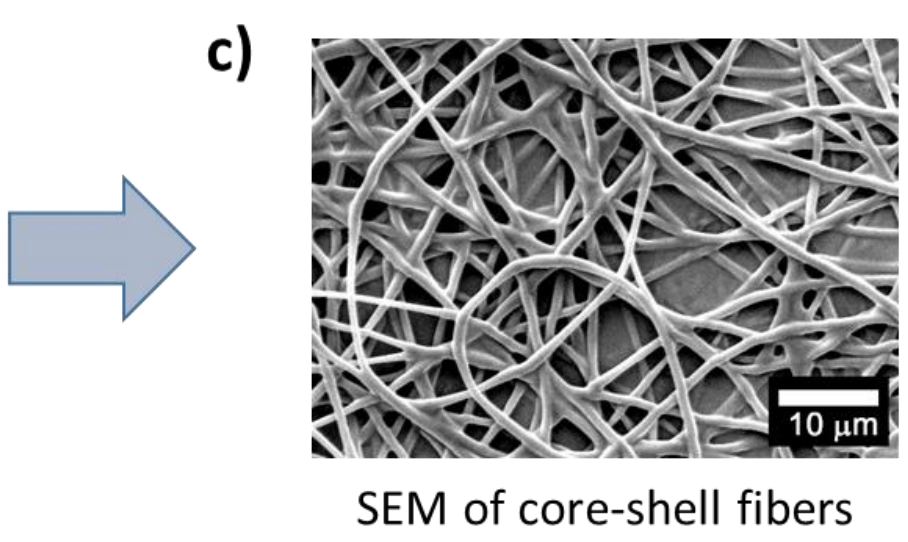

Figure 1. Fabrication of core-shell electrospun fibers: a) schematic of coaxial electrospinning setup, b) schematic of core-shell fibers of PVA (shell, shown in blue) and healing agent (core, shown in green), and c) SEM image of randomly deposited core-shell fibers. 


\section{Clean sandblasted steel}
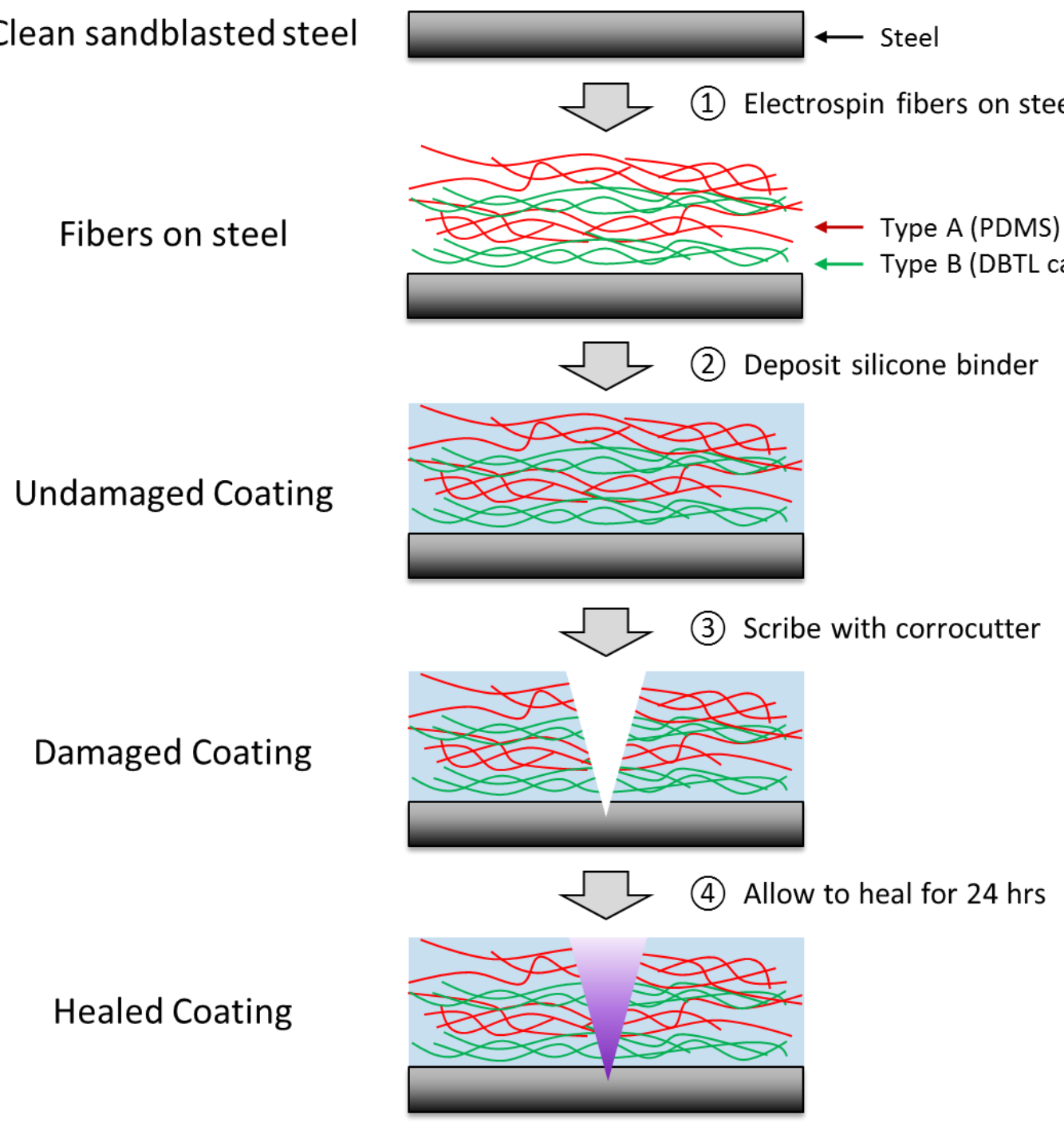

Figure 2. Schematic of coating fabrication process. Samples were prepared on clean sandblasted steel by 1) electrospinning fibers directly onto steel, 2) depositing a silicone binder to affix fibers on steel, 3) scribing the coating with a corrocutter, and 4) allowing the coating to heal for $24 \mathrm{hr}$ before further characterization. 

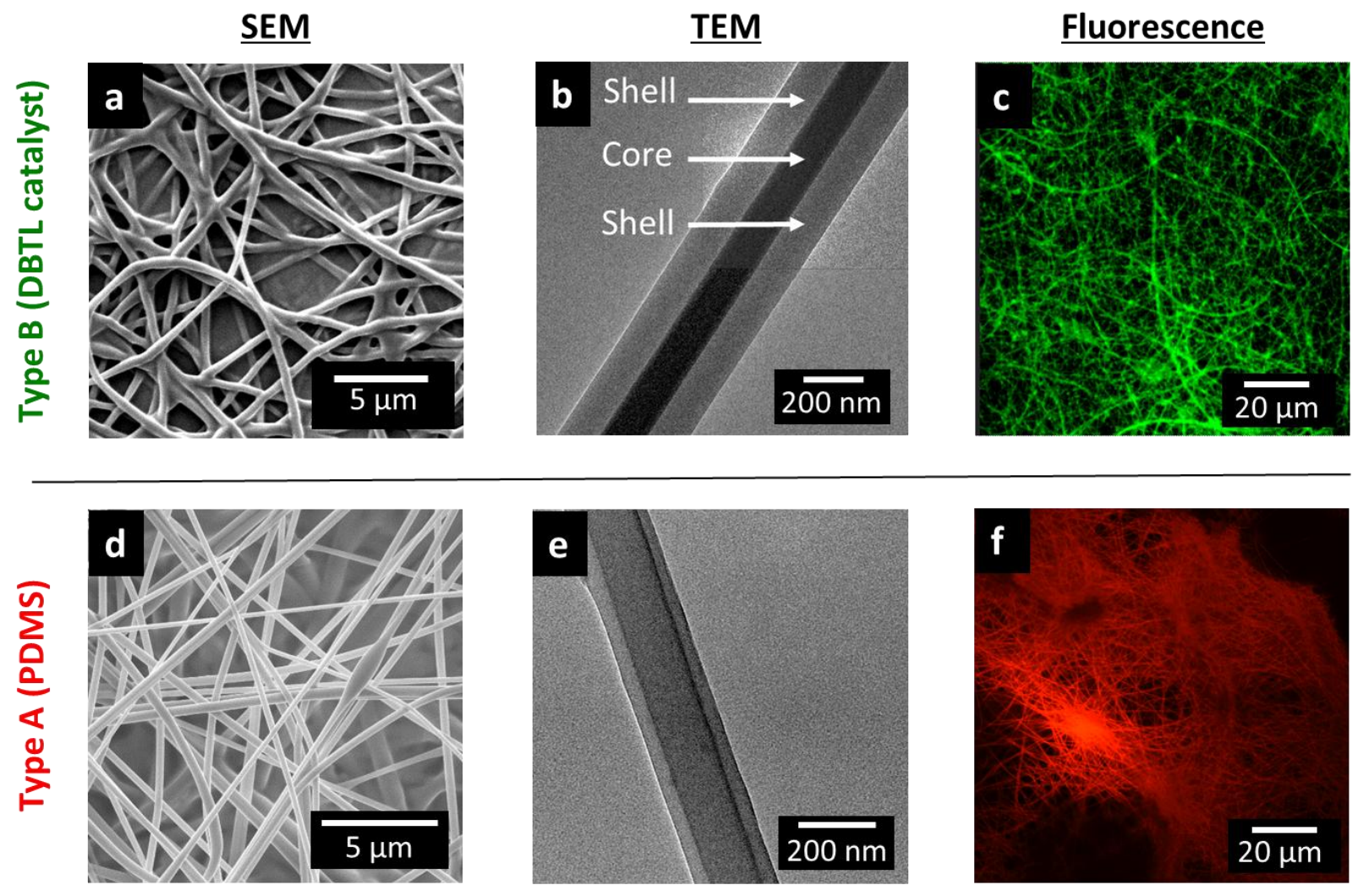

Figure 3. Evidence of core-shell fiber structure: (a) SEM, (b) TEM, and (c) fluorescence microscopy of fibers containing DBTL catalyst core with coumarin 6 fluorescent dye, (d) SEM, (e) TEM, and (f) fluorescence microscopy of fibers containing PDMS core with sudan blue fluorescent dye. 

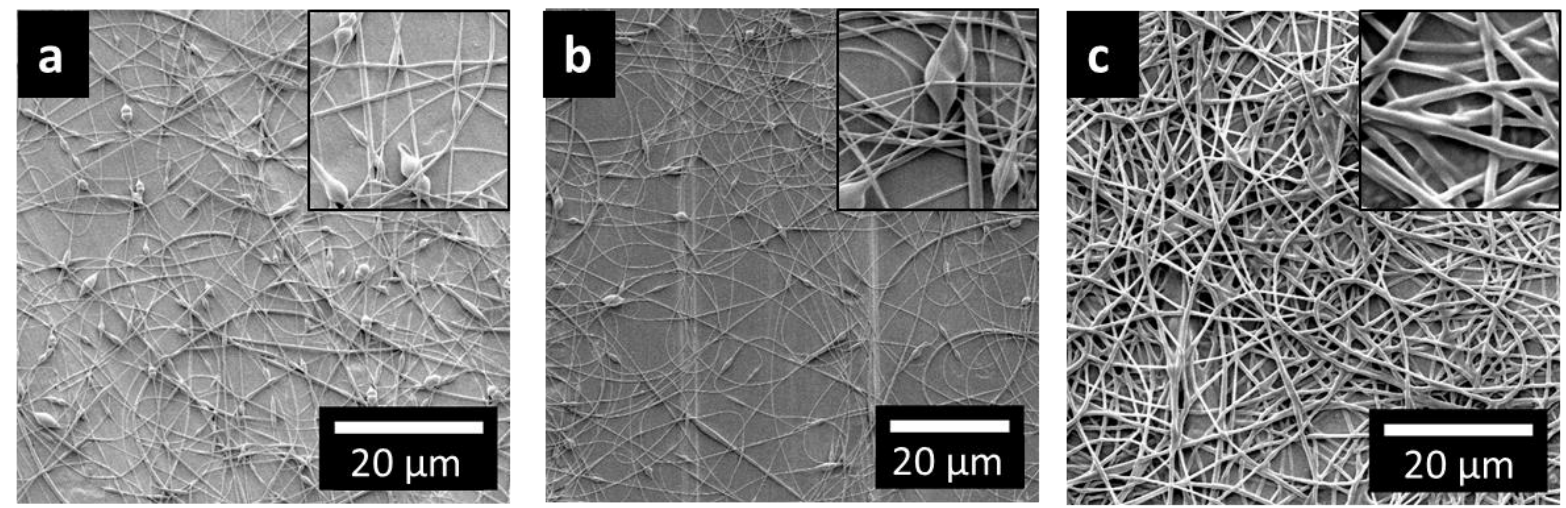

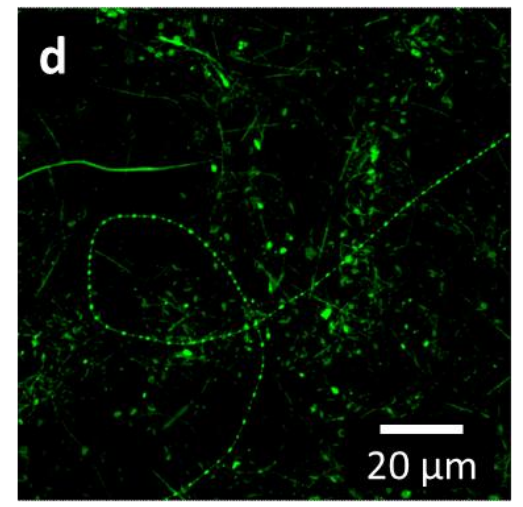

$4 \mu \mathrm{L} / \mathrm{min}$ (shell)

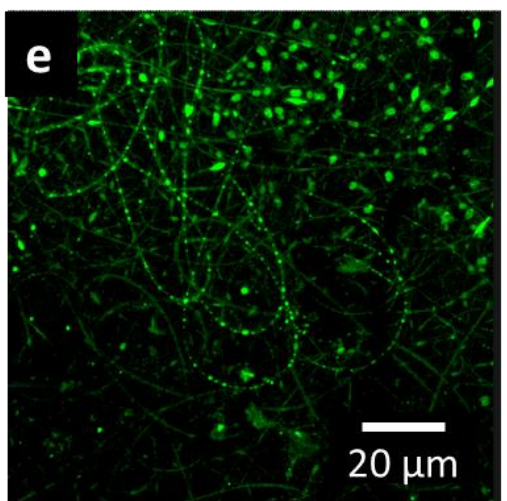

$5 \mu \mathrm{L} / \mathrm{min}$ (shell)

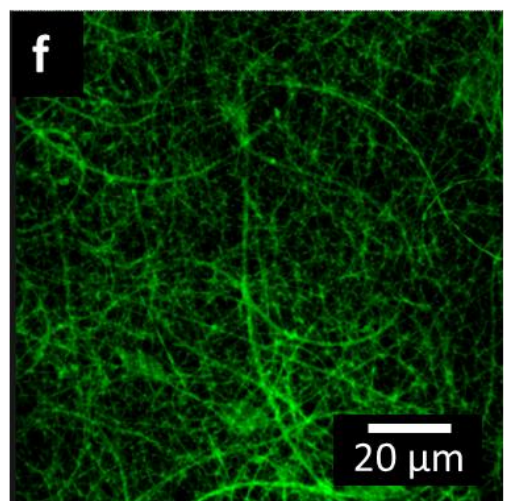

$6 \mu \mathrm{L} / \mathrm{min}$ (shell)

Figure 4. Effect of processing parameters on core-shell morphology. SEM and confocal fluorescence microscopy images of core-shell fibers electrospun at increasing solution pumping rate: (a, d) $4.0 \mu \mathrm{L} / \mathrm{min}$ (shell) and $3 \mu \mathrm{L} / \mathrm{min}$ (core), (b,e) $5.0 \mu \mathrm{L} / \mathrm{min}$ (shell) and $3 \mu \mathrm{L} / \mathrm{min}$ (core), (c, f) $6.0 \mu \mathrm{L} / \mathrm{min}$ (shell) and $3 \mu \mathrm{L} / \mathrm{min}$ (core). Insets in SEM images are shown at 6500x magnification. 


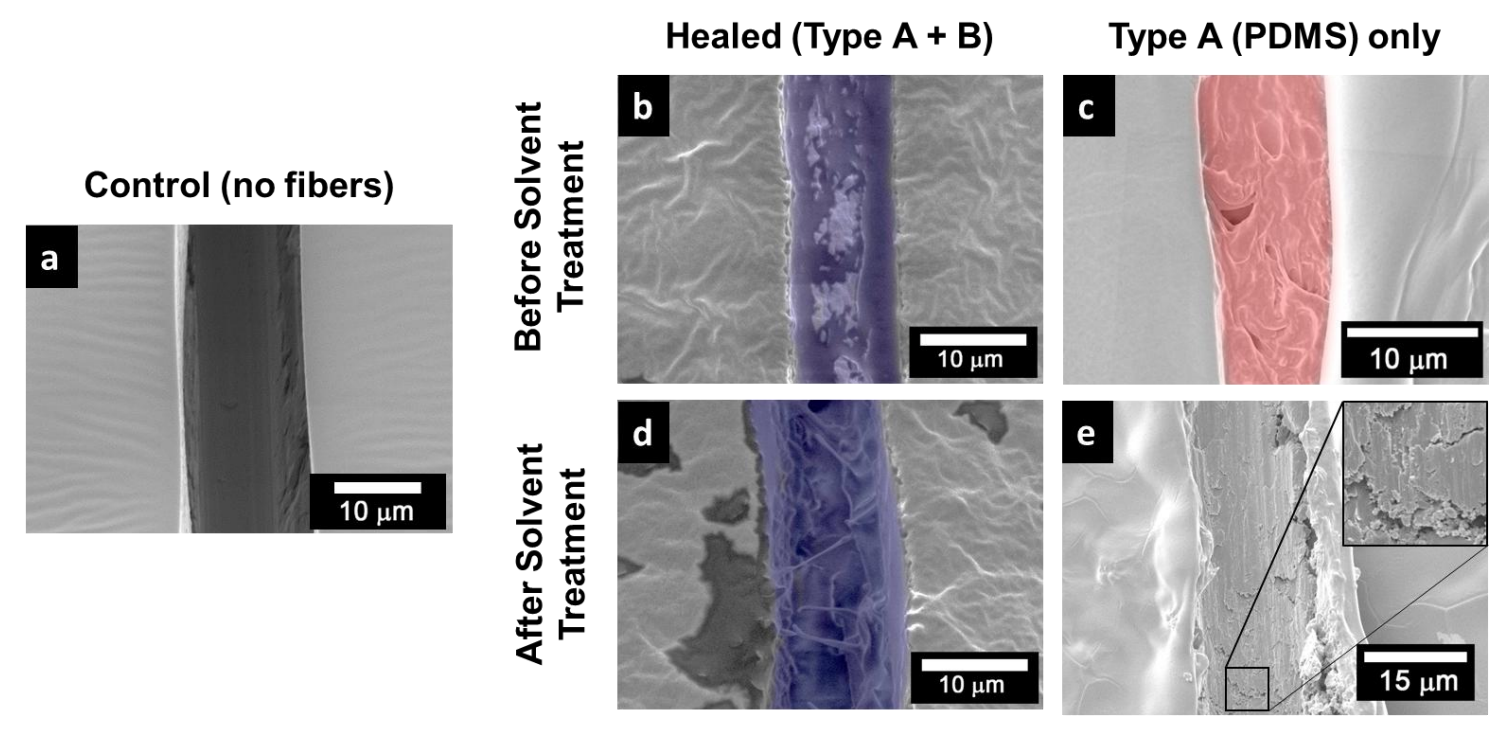

Figure 5. Evidence of released material in the damaged region of the coatings. SEM images of coatings (48 hours after damage) before and after solvent treatment to remove unreacted healing agents. (a) Control coating control coating without self-healing fibers and only silicone binder is shown for comparison. Before solvent treatment images of (b) self-healing coating and (c) type A (PDMS) only control coating. After solvent treatment, (d) the polymerized healing agents are retained in the scribed region and (e) unreacted PDMS is removed showing the underlying steel substrate. 


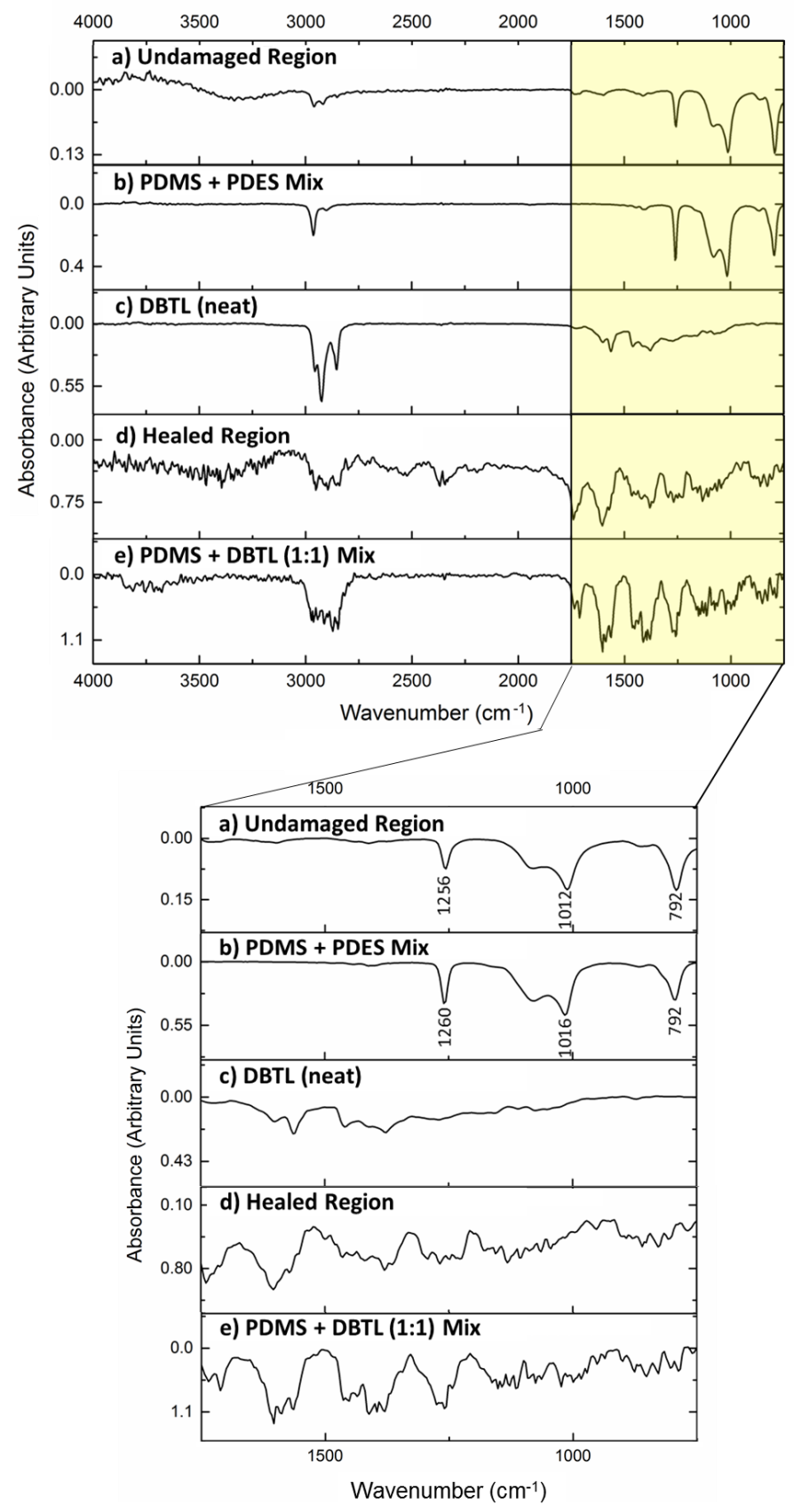

Figure 6. Chemical analysis of damaged region. Summary of FTIR spectra for a) self-healing coating in the undamaged region, b) solution of PDMS and PDES, c) neat DBTL catalyst, d) self-healing coating in the damaged region, and e) mixture of both healing agents (PDMS and DBTL). The full spectral range of the FTIR experiments are shown in (1) and the fingerprint region (2) is expanded for clarity. 


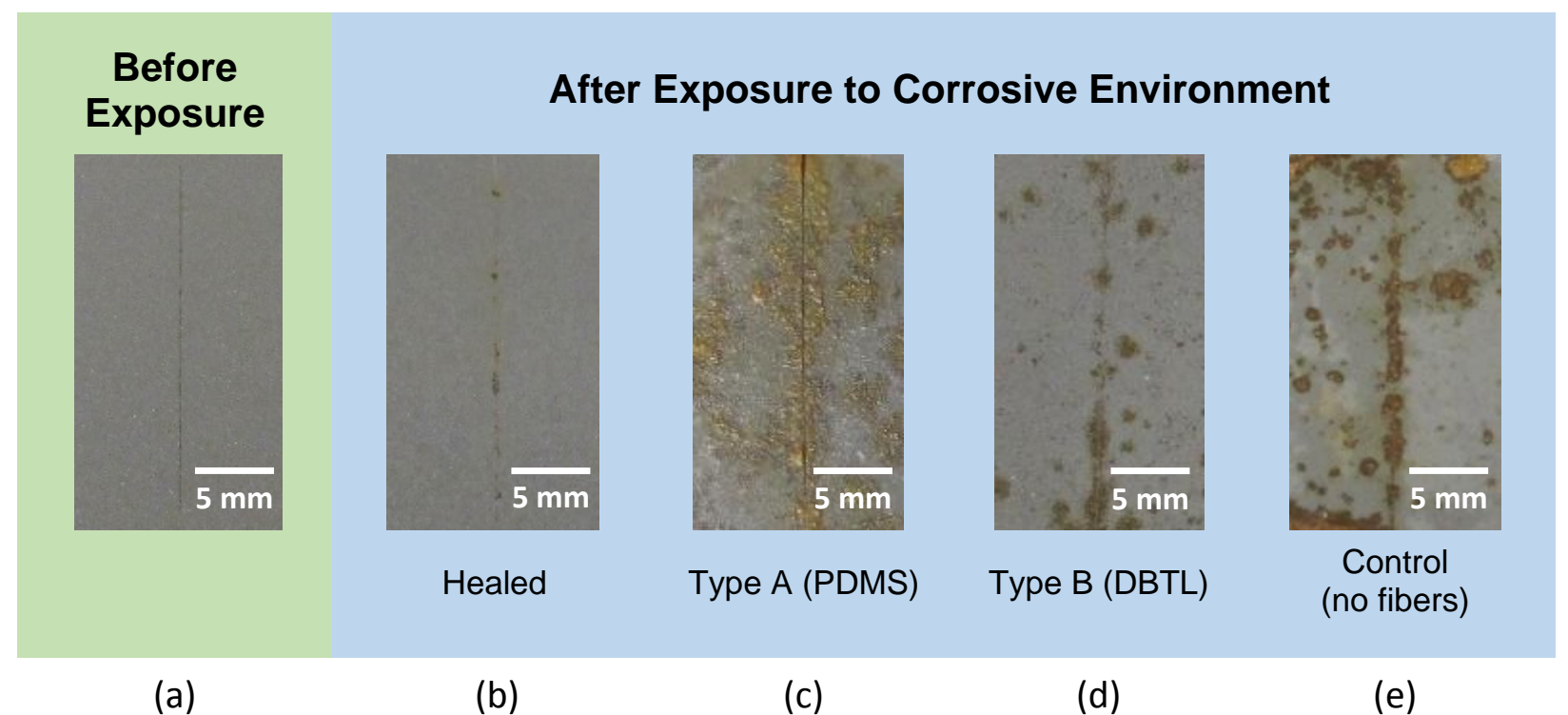

Figure 7. Optical images of healed and control coatings 4 months after exposure to corrosive aqueous salt solution during electrochemical characterization. The healed coating before and after corrosion exposure is similar. However, the control cases of type A (PDMS) fibers only, type B (DBTL catalyst) fibers only, and no fibers (silicone binder only) show obvious signs of corrosion damage. 


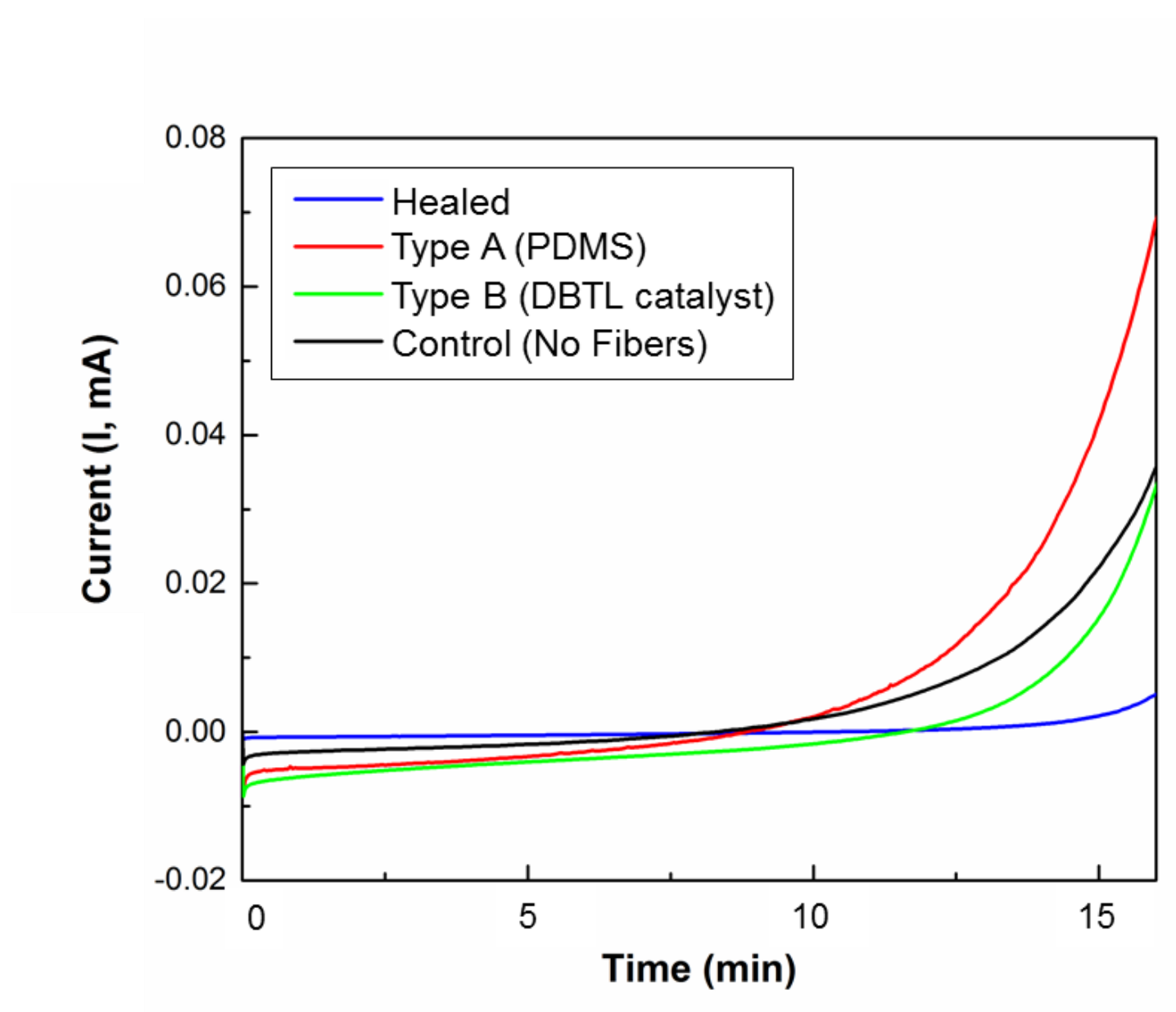

Figure 8. Electrochemical characterization of coating types. Linear polarization sweep plot of current $(I)$ vs. time for coatings containing control with silicone binder only and no fibers (black), type A (PDMS) fibers (red), and type B (DBTL catalyst) fibers (green). The healed coating (blue) has a lower corrosion current than any of the control cases. [Note: Curves are from one representative sample of each coating type]. 
a)

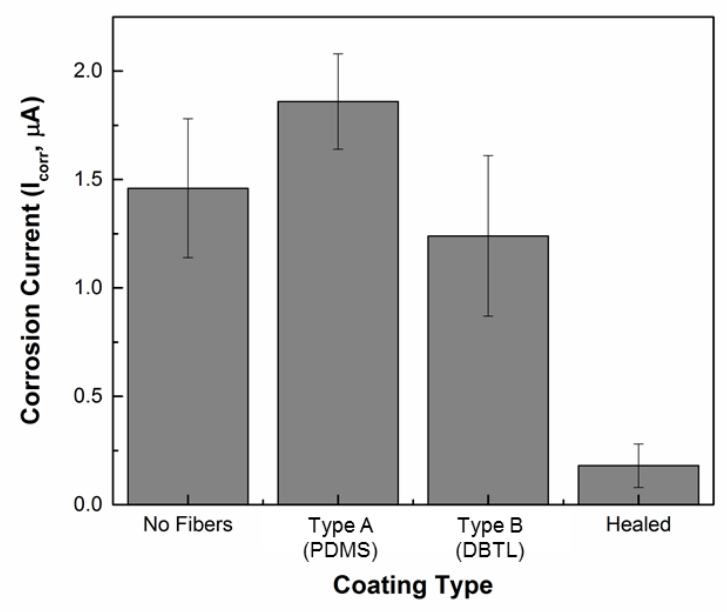

b)

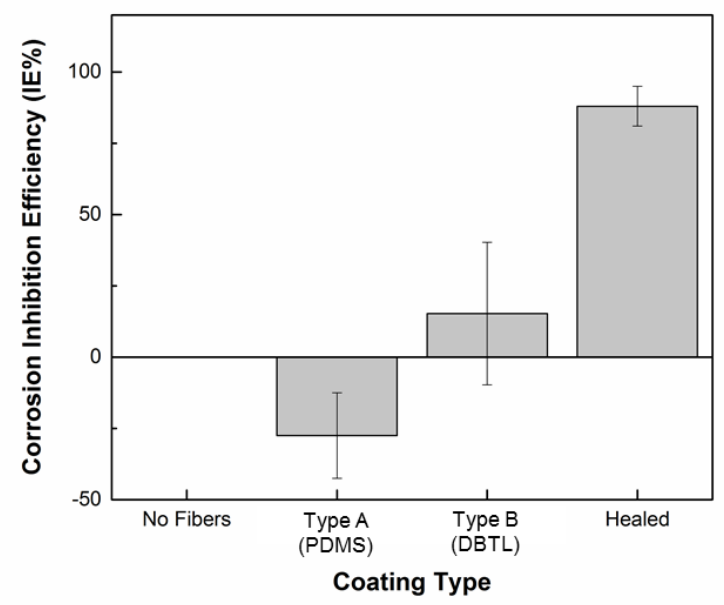

Figure 9. Assessment of corrosion inhibition. Comparison of a) corrosion current $\left(I_{\text {corr }}\right)$ and b) corrosion inhibition efficiency (IE\%) for each coating type: control coating with no fibers, type A (PDMS) fibers only, type B (DBTL catalyst) fiber only, and healed coating containing both type A and B fibers. [Note: vertical error bars represent the standard error from five samples tested]. 


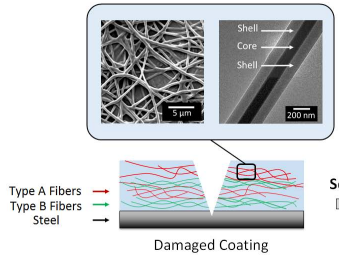

Damaged Coating

Self-heal

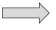

$\overline{5 \mathrm{~mm}}$

Healed

Control

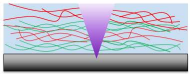

Healed Coating 